\title{
The acute effect of baobab fruit on cognitive performance, cerebral blood flow and blood glucose levels.
}

\section{Abstract}

Baobab fruit contains high levels of phenolic compounds and vitamin C. Previous work has associated these phenolic compounds and vitamin $\mathrm{C}$ with a range of health benefits including improvements in cerebral blood flow and cognition. In vivo, it has been demonstrated that consumption of baobab fruit can reduce the glycaemic response, which may provide a mechanism for cognitive benefits as other research has shown that variations in blood glucose levels can modulate cognitive performance. Preliminary work found that consumption of $15 \mathrm{~g}$ baobab fruit extract significantly improved reaction time but increased number of errors on an executive function task. Taken together it would suggest baobab fruit has the potential to improve cognitive performance that could be attributed to changes in cerebral blood flow and blood glucose levels.

The current study aimed to determine the effect of baobab fruit on cognitive performance, cerebral blood flow and blood glucose levels in a healthy young sample.

This randomised, placebo-controlled, double-blind, counterbalanced-crossover study assessed the effect of $10 \mathrm{~g}$ baobab fruit powder or sugar matched control in 24 healthy participants $(17$ female, 7 male, mean age $=22.91 \mathrm{SD}=3.37$ ). All participants completed the cognitive assessments, a subset of 14 completed the cerebral blood flow and blood glucose assessments $(\mathrm{mean}$ age $=23.21, \mathrm{SD}=2.46)$. Participants completed baseline tasks before consuming a drink containing either $10 \mathrm{~g}$ baobab fruit powder or placebo, there was then a 45-minute absorption period before participants completed cognitive tasks again. Seven days after participants returned and completed the same procedure but consumed the opposite drink. In the 14 participant subset, cerebral blood flow was measured throughout using Near Infrared Spectroscopy (NIRS) and blood glucose was measured before testing, after absorption period and upon completion of post dose

Using the MIXED procedure in SPSS, results showed that after consumption of baobab there was improved accuracy on a sustained attention task and fewer errors on the last repetition of a serial subtraction task. Baobab consumption led to increased blood glucose levels but there was no significant effect on cerebral blood flow.

Results show that, in this sample, $10 \mathrm{~g}$ baobab fruit can improve certain aspects of cognitive performance and increase circulating blood glucose levels, which may explain these improvements. However, there was no significant effect on any cerebral blood flow measures. Future work may wish to explore further glucoregulation activity (in particular insulin response) after baobab consumption as a potential underlying mechanism.

\section{Conflict of Interest}

There is no conflict of interest 